\title{
Optical Fibre Sensors for Monitoring Phase Transitions in Phase Changing Materials
}

\author{
Rahul Kumar \\ Northumbira University, Faculty of Engineering \& Environments, Newcastle, United Kingdom \\ Wei Han \\ Technological University Dublin, wei.han@tudublin.ie \\ Dejun Liu \\ Technological University Dublin, dejun.liu@tudublin.ie
}

See next page for additional authors

Follow this and additional works at: https://arrow.tudublin.ie/engscheleart2

Part of the Electrical and Computer Engineering Commons

\section{Recommended Citation \\ Kumar, R., Han, W. \& Liu, D. (2018). Optical fibre sensors for monitoring phase transitions in phase changing materials. Smart Materials and Structures, vol. 27, no. 10. doi:10.1088/1361-665X/aaddba}

This Article is brought to you for free and open access by the School of Electrical and Electronic Engineering at ARROW@TU Dublin. It has been accepted for inclusion in Articles by an authorized administrator of ARROW@TU Dublin. For more information, please contact arrow.admin@tudublin.ie, aisling.coyne@tudublin.ie, gerard.connolly@tudublin.ie.

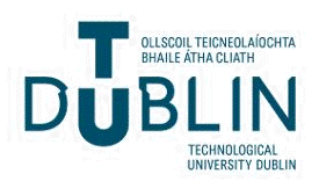




\section{Authors}

Rahul Kumar; Wei Han; Dejun Liu; W. P. Ng; Binns, Richard Binns, Richard; Krishna K. Busawon; Yong Qing Fu; Zabih Ghassemlooy; Chris P. Underwood; Khamid Kh Mahkamov; Jinhui Yuan; Chongxiu Yu; Huazhong Shu; Xing Ao Li; Tuan Guo; Gerald Farrell; Yuliya Semenova; and Qiang Wu 


\section{PAPER}

Optical fibre sensors for monitoring phase transitions in phase changing materials

To cite this article: Rahul Kumar et al 2018 Smart Mater. Struct. 27105021

View the article online for updates and enhancements. 


\title{
Optical fibre sensors for monitoring phase transitions in phase changing materials
}

\author{
Rahul Kumar ${ }^{1,6}$, Wei Han ${ }^{2,6}$, Dejun Liu ${ }^{2}$, Wai Pang $\mathrm{Ng}^{1}$, Richard Binns ${ }^{1}$, \\ Krishna Busawon ${ }^{1}$, Yong Qing Fu ${ }^{1}$, Zabih Ghassemlooy ${ }^{1}$, \\ Christopher Underwood $^{1}$, Khamid Mahkamov ${ }^{1}$, Jinhui Yuan ${ }^{3}$, \\ Chongxiu $\mathrm{Yu}^{3}$, Huazhong Shu ${ }^{4}$, Xing Ao $\mathrm{Li}^{4}$, Tuan Guo ${ }^{5}$, Gerald Farrell ${ }^{2}$, \\ Yuliya Semenova ${ }^{2}$ and Qiang $\mathrm{Wu}^{1}$ (1) \\ ${ }^{1}$ Faculty of Engineering and Environment, Northumbria University, Newcastle Upon Tyne, NE1 8ST, \\ United Kingdom \\ ${ }^{2}$ Photonics Research Centre, Dublin Institute of Technology, Kevin Street, Dublin 8, Ireland \\ ${ }^{3}$ State Key Laboratory of Information Photonics and Optical Communications, Beijing University of Posts \\ and Telecommunications, Beijing, People's Republic of China \\ ${ }^{4}$ Key Laboratory for Organic Electronics \& Information Displays (KLOEID), Institute of Advanced \\ Materials (IAM), School of Materials Science and Engineering (SMSE), Nanjing University of Posts and \\ Telecommunications (NUPT), Nanjing 210023, People's Republic of China \\ ${ }^{5}$ Guangdong Provincial Key Laboratory of Optical Fiber Sensing and Communications, Institute of \\ Photonics Technology, Jinan University, Guangzhou 510632, People's Republic of China
}

E-mail: qiang.wu@northumbria.ac.uk

Received 13 April 2018, revised 26 August 2018

Accepted for publication 30 August 2018

Published 14 September 2018

\begin{abstract}
A platinum coated singlemode-multimode (SM) structure is investigated in this paper as an optical fibre sensor (OFS) to monitor the phase transition of a phase change material (PCM). Paraffin wax has been used as an example to demonstrate the sensor's performance and operation. Most materials have the same temperature but different thermal energy levels during the phase change process, therefore, sole dependency on temperature measurement may lead to an incorrect estimation of the stored energy in PCM. The output spectrum of the reflected light from the OFS is very sensitive to the bend introduced by the PCM where both liquid and solid states exist during the phase transition. The measurement of strain experienced by the OFS during the phase change of the PCM is utilized for identifying the phase transition of paraffin wax between the solid and liquid states. The experimental results presented in this paper show that the OFS with a shorter multimode fibre section has better performance for monitoring the phase transition of paraffin wax with a measured phase transition temperature range of $41.5^{\circ} \mathrm{C}-$ $57.7^{\circ} \mathrm{C}$ for the SM based OFS with a $5 \mathrm{~mm}$ long multimode fibre section.
\end{abstract}

Keywords: optical fibre sensor, phase change material, phase change monitoring, SMS structure, FBG

(Some figures may appear in colour only in the online journal)

\section{Introduction}

Solid-liquid phase change materials (PCMs) have been widely used in latent heat thermal storage systems for heat pumps [1-3], solar engineering [4], and spacecraft thermal

\footnotetext{
6 These authors have equal contributions to this paper.
}

control [5]. A simple and common application of PCM can be seen in thermally insulated water bottles, where the PCM surrounded by water melts and is used to store the heat from the hot water. The stored heat is then released back to the water at a slower rate as the PCM solidifies so as to maintain a desired water temperature over a longer period of time than would be the case if a simple insulator was used instead of a 
PCM. There are a large number of PCMs, which melt and solidify over a wide range of temperatures, which are attractive for a wide number of applications [5, 6]. The practical realization of an efficient PCM-based thermal energy management system requires an in-depth understanding of the PCM behaviour during melting and solidification stages. Solidification can occur below the expected phase transition temperature, a phenomenon referred to as under-cooling [7]. Temperature measurements are widely used to measure the amount of stored thermal energy in PCMs. However, in many cases at the solid-liquid phase (SLP) change state, most materials have the same temperature but different energy levels. For example, at $0{ }^{\circ} \mathrm{C}, 1$ gram of liquid water has 333.55 Joules more energy than that of the solid ice. Therefore, sole dependency on temperature measurement alone may lead to an incorrect estimation of the stored energy in the PCM, particularly in a large PCM-based energy storage system. To address this issue, several techniques such as differential scanning calorimetry (DSC) [8] and differential thermal analysis (DTA) [9] have been proposed to determine the phase state of PCM. However, these techniques are complex to operate which usually requires a skilled technician to carry out measurement and costly. It is hence necessary to develop a new technique to monitor the SLP change by determining the start and end points of phase changes, a measurement which cannot be undertaken by traditional temperature sensors. One possible option is the use of optical fibre sensor (OFS) technology.

Due to the advantages of compact size, immunity to electromagnetic interference and remote sensing capabilities [10], OFSs have been widely used in many areas such as automotive, chemical industry, aircraft, medical diagnosis areas, etc [11]. There are different types of OFSs including fibre Bragg grating (FBG) sensors [12-14], long period grating sensors $[15,16]$, photonic crystal fibre (PCF) sensors [17, 18], Fabry-Perot interferometer sensors [19], and singlemode-multimode-singlemode (SMS) fibre sensors $[20,21]$. Among these sensors, SMS sensors, which possess a simple fabrication process and low cost with high sensitivity, have attracted wide research interest in recent years for measurements of strain $(\varepsilon)[22,23]$, temperature [24-26], refractive index [27, 28], and chemical concentrations [29]. Our previous investigations demonstrated that an SMS sensor is very sensitive to even minor bending forces applied to it [30]. In a PCM at the SLP change point, small local variations in the state of material surrounding the fibre result in slightly different forces being applied to different locations on the SMS fibre sensor, since these local variations occur on a spatial scale similar in size to the dimensions of the SMS sensor. The result is that micro bends occur along the length of the SMS sensor and in turn this leads to a significant wavelength shift $\Delta \lambda$ in the output spectrum of the SMS fibre sensor. In this paper we experimentally investigate an SMS fibre based sensor structure as a better and more accurate means to monitor the phase change $(\delta \phi)$ of a PCM between solid and liquid states. The well-known PCM paraffin wax (327204 Aldrich) having a melting point between $53{ }^{\circ} \mathrm{C}-$ $57^{\circ} \mathrm{C}$ is used in this experiment as the host material because

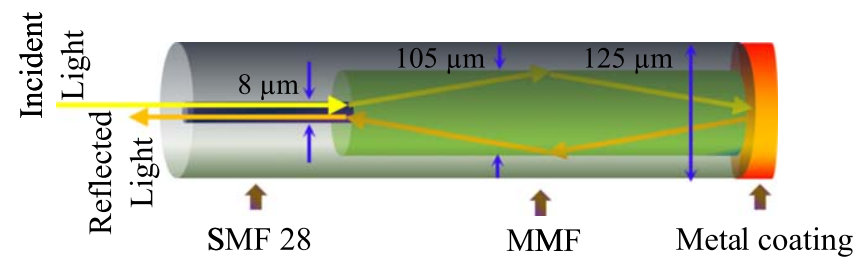

Figure 1. Schematic diagram of reflective SM optical fibre structure.

of its low cost and availability. In future, the proposed sensor can be used as a cheaper alternate for real time phase state monitoring of other PCM, such as paraffin/polyethylene blend [31], polyethylene/paraffin blend [32] and erythritol [3], etc used in thermal storage system.

The paper is organised as follows: section 2 describes the experimental setup along with the fabrication and operating principle of a reflective singlemode-multimode (SM) structure working as an optical fibre sensor structure to identify different phase states of the paraffin wax. This structure optically works as an SMS fibre structure but is configured as an endpoint sensor and is used here since measurement in a PCM environment is best suited to a probe type sensor. Section 3 describes the results, based on which the sensor was optimised for better accuracy. Finally, section 4 presents our conclusions.

\section{Experimental set-up}

In this experiment, two reflective SM fibre sensors with different multimode fibre (MMF) of length 5 and $10 \mathrm{~mm}$ were fabricated in order to investigate the influence of the MMF length on the measurement accuracy when monitoring the $\delta \phi$ point of the paraffin wax as shown in figure 1 . The reflective SM structure is fabricated by fusion splicing a short length of MMF (AFS105/125Y) with a conventional SMF (SMF28), and coating the unconnected MMF end with a thin layer of platinum (10 $\mathrm{nm}$ thickness). Note that, in principle both the reflective and the conventional SMS fibres have the same transmission principle. Light propagates along the SMF and on entering the MMF will excite multiple modes within its core. Since the MMF end is coated with the platinum coating, the propagating light will be reflected back into the MMF fibre and in turn is coupled back into the SMF. The interference between multiples modes within the MMF results in transmission dips in the output spectral response, which depend on the surrounding environment, i.e., local temperature $(T)$, longitudinal strain $(\varepsilon)$ and the fibre bending.

The schematic diagram of the experimental setup for measuring $\delta \phi$ of the paraffin wax is shown in figure 2. A glass rod is used to fix both the optical fibre sensor and a thermocouple as close as possible ( $<2 \mathrm{~mm}$ apart), with the rod and attachments then immersed in a beaker containing $250 \mathrm{ml}$ of paraffin wax sample. A broadband light source (SLD1005S) is used to launch light into the optical sensor via an optical circulator (OC). The reflected light from the sensor is observed with an optical spectrum analyser (OSA, Yokogawa AQ6370D) via the OC in order to analyse its spectral 


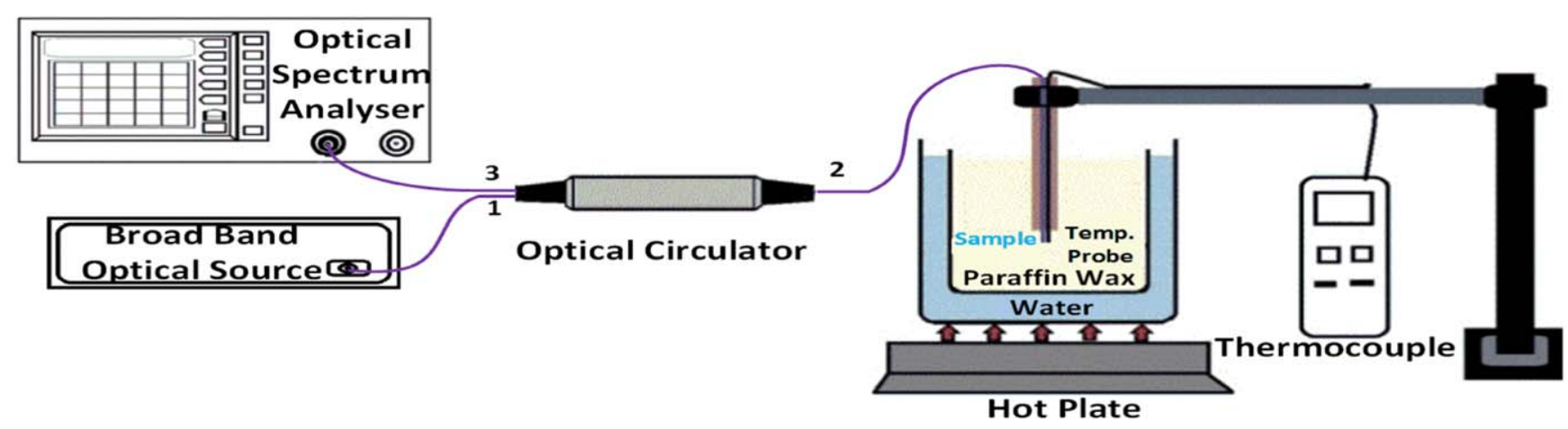

Figure 2. Schematic diagram of experimental setup for measuring phase change $(\delta \phi)$ of paraffin wax.

response. The OSA is programmed to automatically record dip wavelength (maximum dip) every 2 min over a wide wavelength range from 1450 to $1650 \mathrm{~nm}$. A thermocouple (RS 206-3738, K type, Accuracy $\pm 0.2 \%$ reading error $+1{ }^{\circ} \mathrm{C}$ ) is used as a temperature reference from which the temperature readings are recorded every $2 \mathrm{~min}$ to correlate with the reading from OSA. The heating and cooling conditions were maintained to be the same by using a water bath to gradually increase or decrease the temperature. In addition, while heating the hot plate the temperature was set to a level so that the time required to heat the wax from its solid state to the liquid state is the same as that required to cool the wax back to its solid state.

\section{Result and discussion}

Figures 3(a) and (b) show the measured $\Delta \lambda$ as a function of temperature $\left(30.0^{\circ} \mathrm{C}-70.0^{\circ} \mathrm{C}\right)$ of the platinum coated SM fibre structures with $5 \mathrm{~mm}$ and $10 \mathrm{~mm}$ long MMF, respectively. Note that, to validate the reliability and repeatability of the proposed sensor two sets of measurements were carried out. The temperature $T$ of the paraffin wax was measured with the thermocouple.

As shown in figure 3(a), there are two temperature ranges (i.e., $30.0^{\circ} \mathrm{C}<T<41.5^{\circ} \mathrm{C}$ and $57.7^{\circ} \mathrm{C}<T<70.0^{\circ} \mathrm{C}$ ) where the paraffin wax is in a solid and liquid states respectively and where the wavelength responses are fairly linear but with different slopes. The result indicates that the shift in the spectral dip within the temperature ranges used is mainly due to the temperature-induced strain on the sensor immersed in solid and liquid paraffin, respectively. Of most interest is the temperature range $41.5{ }^{\circ} \mathrm{C}<T<57.7^{\circ} \mathrm{C}$, where both solid and liquid states exist simultaneously and where the stress applied along the length of the fibre sensor is not uniform which in turn induces random micro-bends in the SM fibre sensor, thus resulting in a random wavelength shift with temperature. Note that, the abrupt nature of the changes in the spectral wavelength is likely due to the random lateral force applied to the sensor by the solid-liquid mixed phase states of the wax, which leads to an abrupt micro-bending state at a random position with a random radius along the MMF section of the fibre sensor. Figure 3(a) also shows a good agreement in terms of the temperature range within which the phase change takes place for the measurements for both cycles 1 and 2 (i.e., heating and cooling $T$ ) as well as a similar response for each cycle. Note that, for all the measurements taken at $T>57.7^{\circ} \mathrm{C}$ the wavelength profiles are the same. This is because with the wax in the liquid state and the forces acting upon the sensor are small and homogenous, resulting in the absence of micro bending of the SM fibre sensor. However, for $T<41.5^{\circ} \mathrm{C}$ the wax is in a solid state resulting in micro bending of the sensor. Note that, the micro bending state appears again when the wax cools down from the liquid to the solid phase state, thus resulting in a different but linear $\Delta \lambda$ as a function of $T$ (i.e., for all four sets of measurements for $T<41.5^{\circ} \mathrm{C}$ ). For $T<41.5^{\circ} \mathrm{C}$, the wax is fully solidified and hence the temperature induced micro bending in the SM fibre sensor is relatively small, thus resulting in a linear wavelength response. Similar changes in the wavelength with temperature are also observed for the reflective SM structure with a $10 \mathrm{~mm}$ long MMF, see figure 3(b). The primary difference between the responses observed for the sensors with 5 and $10 \mathrm{~mm}$ long MMFs is in the temperature range where random fluctuations of the wavelength are clearly seen (i.e., indicating the $\delta \phi$ of the paraffin wax), which are $41.5^{\circ} \mathrm{C}-57.7^{\circ} \mathrm{C}$ and $37.8^{\circ} \mathrm{C}-$ $57.7^{\circ} \mathrm{C}$ for $5 \mathrm{~mm}$ and $10 \mathrm{~mm}$ long MMFs, respectively. This is because the sensor's area immersed into the wax is larger because of the longer MMF, thus higher temperature difference $\Delta T$ appears across the MMF section compared with the shorter length MMF, since the temperature distribution in the wax is not uniform. Note, that a higher $\Delta T$ along the sensor's length will introduce more micro bending in the MMF section, thus resulting in random $\Delta \lambda$ changes over a much larger temperature range read by the thermocouple, which is not desirable. The purpose of the sensor is to monitor $\delta \phi$ at a single point with no influence of other points in the wax. The undesired temperature influence from the adjoining layers of the wax for the shorter length MMF (i.e., $5 \mathrm{~mm}$ ) sensor is rather small in comparison with the longer length MMF (i.e., $10 \mathrm{~mm}$ ) sensor. Therefore, we can conclude that the sensor with a $5 \mathrm{~mm}$ long MMF offers improved measurement accuracy within the phase change state $\left(41.5^{\circ} \mathrm{C}<T<57.7^{\circ} \mathrm{C}\right)$ compared to that of $10 \mathrm{~mm} \mathrm{MMF}$ sensor.

Figure 4 shows the measured $T$ and the wavelength shift as a function of time for the paraffin wax within the temperature range $30.0^{\circ} \mathrm{C}<T<73.0^{\circ} \mathrm{C}$ for both heating and cooling cycles. Also depicted are the typical spectral 

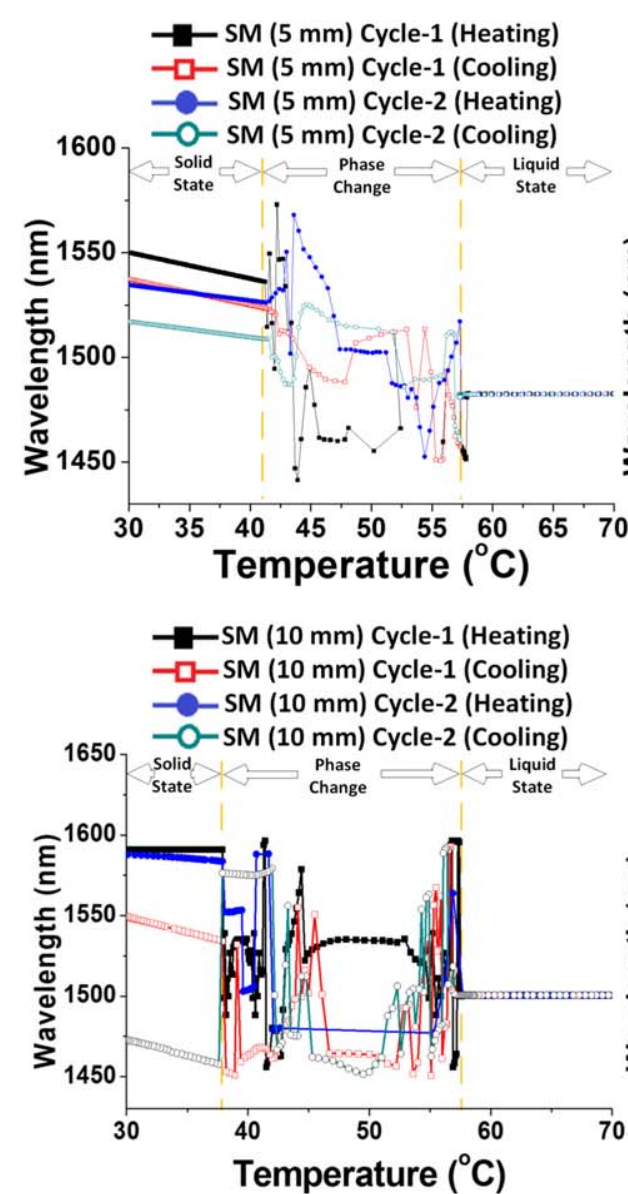

Zoomed solid state - phase change interface

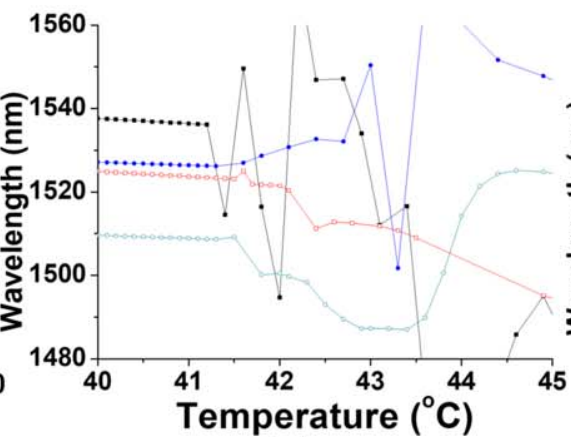

(a)

Zoomed solid state - phase change interface

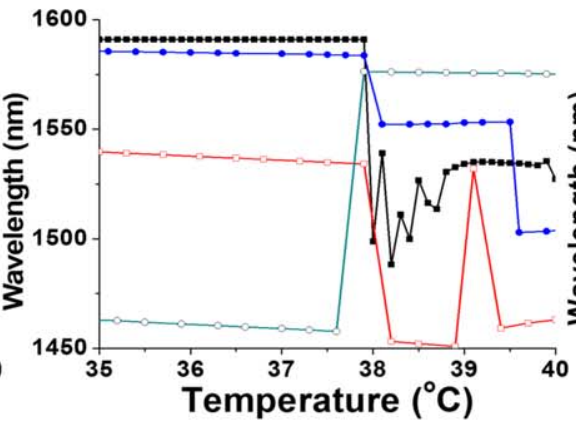

(b)
Zoomed phase change - liquid state interface

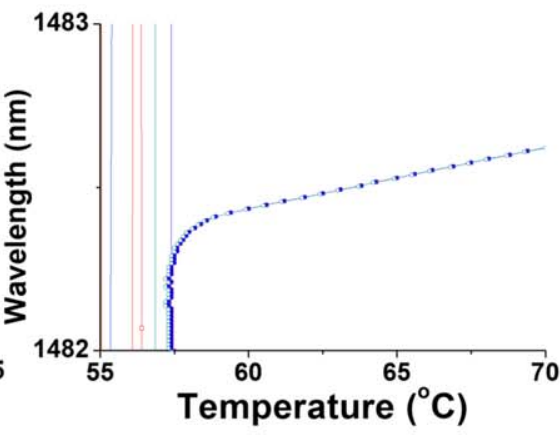

Figure 3. Measured wavelength shift $(\Delta \lambda)$ versus the temperature (T) for the platinum coated SM structure with MMF length of: (a) 5 mm, and (b) $10 \mathrm{~mm}$. Two sets of measurements are taken by heating and then cooling the paraffin wax over a temperature range of $30.0{ }^{\circ} \mathrm{C}-70.0{ }^{\circ} \mathrm{C}$.

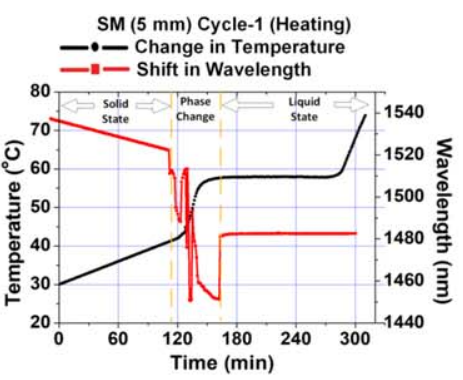

SM (5 mm) Cycle-1 (Cooling)

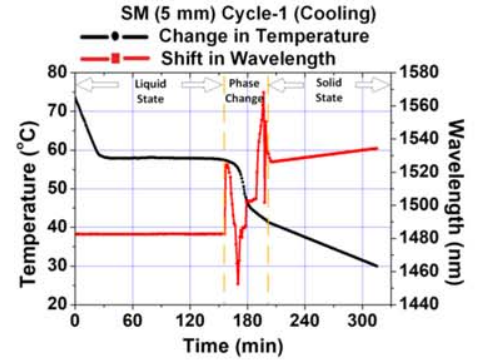

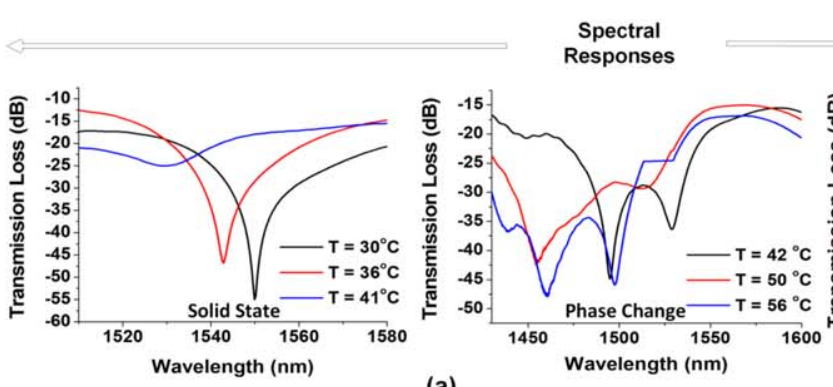

(a)
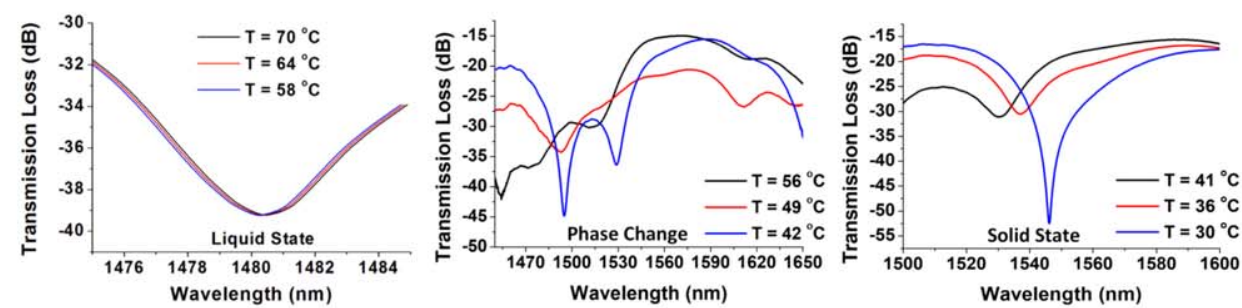

(b)

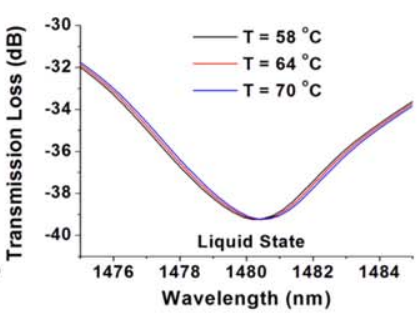

Figure 4. Comparison between the measured temperature $(T)$ and the wavelength shift $(\Delta \lambda)$ as a function of time $(t)$ for the paraffin wax over $30{ }^{\circ} \mathrm{C}<T<73{ }^{\circ} \mathrm{C}$ for: (a) heating, and (b) cooling. The typical spectral response for the reflective SM sensor with a $5 \mathrm{~mm}$ long MMF during different phase state of paraffin wax are also presented. 

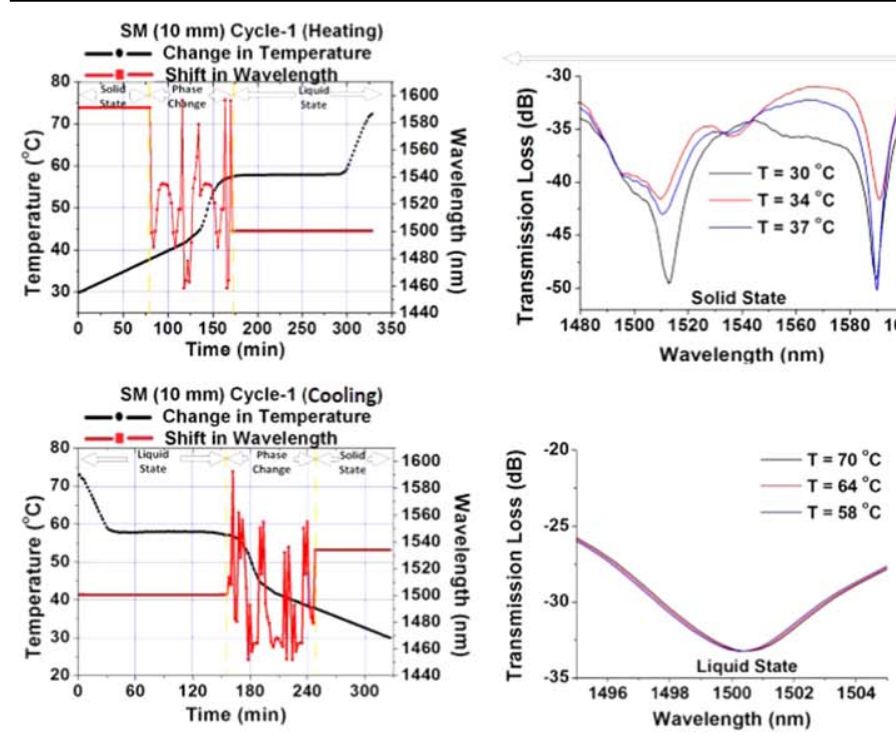
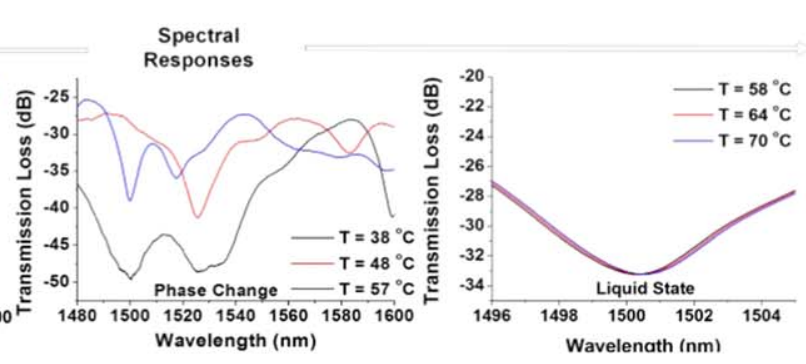

(a)
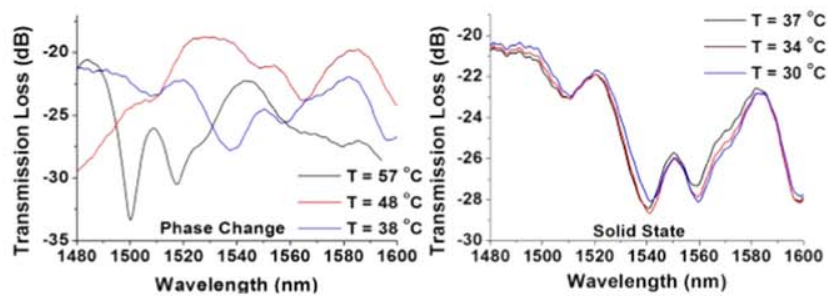

(b)

Figure 5. Comparison between the measured temperature $(T)$ and the wavelength shift $(\Delta \lambda)$ obtained using a reflective SM sensor with a $10 \mathrm{~mm}$ long MMF, as a function of time $(t)$ for the paraffin wax over $30.0^{\circ} \mathrm{C}<T<73.0^{\circ} \mathrm{C}$ for: (a) heating, and (b) cooling cycles.

responses for the reflective SM sensor with a $5 \mathrm{~mm}$ long MMF. As shown in figure 4(a) during the continuous heating process $T$ increases linearly for up to $t=120 \mathrm{~min}$ and then changes rapidly for the next 43 min reaching a steady state value of $\sim 57.7^{\circ} \mathrm{C}$ at $t=163 \mathrm{~min}$. Following this, $T$ continues to increase in a linear manner. The first rise in $T$ is because the solid wax transfers heat with a relatively low thermal conductivity, which results in a significant $\Delta T$ between the bottom of the wax (located near the heat source) and the measured point. After $120 \mathrm{~min}$, the wax enters the phase change state where both liquid and solid will coexist, which significantly increases the thermal conductivity and consequently the abrupt changes in temperature. The constant $T$ during $163<t<273 \mathrm{~min}$ is due to the liquid and solid in the container having the same $T$, where the additional heat energy absorbed by the wax is used to change the phase state from solid to liquid rather than alter the temperature. Beyond $t>273$ min all the wax has changed from solid to liquid and the absorbed heat begins to increase the wax temperature. The spectral response of the reflective SM sensor with the $5 \mathrm{~mm}$ long MMF display a random spectral pattern with no linear relationship in terms of the spectral width, intensity, or number of spectral dips with the changes in $T$, for $121<t<174 \mathrm{~min}$ and $41.4^{\circ} \mathrm{C}<T<57.7^{\circ} \mathrm{C}$. This is because of the aforementioned paraffin wax induced random micro-bending state of the SM sensor due to simultaneous existence of solid and liquid phase states of the wax in the SM sensor area. Figure 4(b) shows the opposite response for both $T$ and $\Delta \lambda$ to that of figure 4(a) for the case when the paraffin wax is cooling. The random spectral response within the time range $156<t<205 \mathrm{~min}$ is consistent with the simultaneous existence of solid and liquid phase states over the temperature range $41.5^{\circ} \mathrm{C}<T<57.7^{\circ} \mathrm{C}$, for the cooling cycle.

Figure 5 shows the measured temperature and the wavelength shift observed using a reflective SM sensor with a
$10 \mathrm{~mm}$ long MMF, as a function of time for the paraffin wax for $30.0^{\circ} \mathrm{C}<T<73.0^{\circ} \mathrm{C}$ for heating and cooling cycles. Both the time and temperature follow the same relationship as explained above for the reflective SM sensor with a $5 \mathrm{~mm}$ long MMF. The spectral response of the reflective SM sensor with a $10 \mathrm{~mm}$ long MMF displays a random wavelength variation in a wider temperature range $\left(37.8{ }^{\circ} \mathrm{C}<T<57.7^{\circ} \mathrm{C}\right)$ for $80<t<172 \mathrm{~min}$ while heating the paraffin wax, in comparison to the reflective SM with $5 \mathrm{~mm}$ long MMF. This is because during the heating and cooling process, the temperature distribution within the paraffin wax sample is not uniform. The longer length $(10 \mathrm{~mm})$ MMF is exposed to a larger area within the paraffin wax hence suffers larger temperature variation along the $10 \mathrm{~mm}$ MMF section compared to that of $5 \mathrm{~mm}$ MMF. However, the reference thermocouple only measures temperature at one point, resulting in a recorded random wavelength variation within a wider temperature range. The random spectral response within the time range of $156<t<250 \mathrm{~min}$ suggests simultaneous existence of solid and liquid phase states at $37.9^{\circ} \mathrm{C}<T<57.6{ }^{\circ} \mathrm{C}$, while cooling the paraffin wax.

A range of measured value for $T$ for different phase states of the paraffin wax for the two cycles of heating and cooling the paraffin wax for the sensor with 5 and $10 \mathrm{~mm}$-long MMFs are given in table 1 .

From the data presented in table 1, we can clearly conclude that the start and end points of the phase change are $41.5^{\circ} \mathrm{C}$ and $57.7^{\circ} \mathrm{C}$ respectively for the $5 \mathrm{~mm}$-long MMF sensor, which is smaller than that $\left(37.8^{\circ} \mathrm{C}\right.$ and $\left.57.7^{\circ} \mathrm{C}\right)$ for the $10 \mathrm{~mm}$-long MMF sensor, showing a better measurement accuracy for shorter MMF sensor. The complete melting point of paraffin wax $\left(57.7^{\circ} \mathrm{C}\right)$ as observed from the proposed sensor contest with the melting point of paraffin wax described in its data sheet. 
Table 1. Measured temperature $(T)$ value for different phase states of the paraffin wax for the sensor with 5 and $10 \mathrm{~mm}$ long MMFs.

\begin{tabular}{|c|c|c|c|c|c|c|c|c|c|}
\hline \multirow[b]{5}{*}{ S. No } & \multirow[b]{5}{*}{ Phase } & \multicolumn{8}{|c|}{ Temperature $\left({ }^{\circ} \mathrm{C}\right)$} \\
\hline & & \multicolumn{8}{|c|}{ Platinum coated SM structure } \\
\hline & & \multicolumn{4}{|c|}{$5 \mathrm{~mm}$ of $\mathrm{MMF}$} & \multicolumn{4}{|c|}{$10 \mathrm{~mm}$ of $\mathrm{MMF}$} \\
\hline & & \multicolumn{2}{|c|}{ Cycle-1 } & \multicolumn{2}{|c|}{ Cycle-2 } & \multicolumn{2}{|c|}{ Cycle-1 } & \multicolumn{2}{|c|}{ Cycle-2 } \\
\hline & & Heating & Cooling & Heating & Cooling & Heating & Cooling & Heating & Cooling \\
\hline 1 & Solid state & $30-41.4$ & $30-41.5$ & $30-41.3$ & $30-41.5$ & $30-37.8$ & $30-37.9$ & $30-37.9$ & $30-37.6$ \\
\hline 2 & Phase change $\delta \phi$ & $41.4-57.7$ & $41.5-57.7$ & $41.3-57.7$ & $41.5-57.8$ & $37.8-57.7$ & $37.9-57.6$ & $37.9-57.7$ & $37.6-57.7$ \\
\hline 3 & Liquid state & $57.7-70.0$ & $57.7-70.0$ & $57.7-70$ & $57.8-70$ & $57.7-70$ & $57.6-70.0$ & $57.7-70.0$ & $57.1-70.0$ \\
\hline
\end{tabular}

\section{Conclusion}

In this paper, a reflective SM structure was proposed for measuring the different phase state of the paraffin wax. During the phase change of the paraffin wax both solid and liquid states existed simultaneously, which result in random lateral forces applied onto the SM structure, thus introducing the abrupt micro-bending in the sensor. The micro-bending of the reflective SM sensor introduced an abrupt wavelength shift in the spectral response of the sensor. Based on the aforementioned principle, the proposed sensor was able to detect the start and end points of phase change of the material. The experimental results presented showed that sensor with a shorter length of MMF offered improved measurement accuracy compared to the longer length MMF.

\section{Acknowledgments}

This work was support by $\mathrm{PhD}$ studentship supported by Faculty of Engineering and Environment, Northumbria University; the National Natural Science Foundation for Excellent Youth Foundation of China (No. 61722505); the Key Program of Guangdong Natural Science Foundation (No. 2018B030311006); State Key Laboratory of Advanced Optical Communication Systems and Networks, Shanghai Jiao Tong University, China.

\section{ORCID iDs}

Qiang Wu (iD https://orcid.org/0000-0002-2901-7434

\section{References}

[1] Lizana J, Chacartegui R, Barrios-Padura A and Ortiz C 2017 Advanced low-carbon energy measures based on thermal energy storage in buildings: a review Renew. Sustain. Energy Rev. 82 3705-49

[2] Zhu N, Hu P, Xu L, Jiang Z and Lei F 2014 Recent research and applications of ground source heat pump integrated with thermal energy storage systems: a review Appl. Therm. Eng. 71 142-51
[3] Underwood C, Shepherd T, Bull S and Joyce S 2018 Hybrid thermal storage using coil-encapsulated phase change materials Energy Build. 159 357-69

[4] Islam M P and Morimoto T 2017 Advances in low to medium temperature non-concentrating solar thermal technology Renew. Sustain. Energy Rev. 82 2066-93

[5] Arshad A, Ali H M, Khushnood S and Jabbal M 2018 Experimental investigation of PCM based round pin-fin heat sinks for thermal management of electronics: effect of pinfin diameter Int. J. Heat Mass Transfer 117 861-72

[6] Wu Y, Chen C, Jia Y, Wu J, Huang Y and Wang L 2018 Review on electrospun ultrafine phase change fibers (PCFs) for thermal energy storage Appl. Energy 210 167-81

[7] Zhou Y, Wu J, Wang R and Shiochi S 2007 Energy simulation in the variable refrigerant flow air-conditioning system under cooling conditions Energy Build. 39 212-20

[8] Kumar S, Agrawal K M, Khan H U and Sikora A 2004 Study of phase transition in hard microcrystalline waxes and wax blends by differential scanning calorimetry Pet. Sci. Technol. 22 337-45

[9] Delgado G E, Sierralta N, Quintero M, Quintero E, Moreno E, Flores-Cruz J A and Rincon C 2018 Synthesis, structural characterization and differential thermal analysis of the quaternary compound $\mathrm{Ag}_{2} \mathrm{MnSnS}_{4}$ Rev. Mex. Física 64 216-21

[10] Zhao Y, Li X-G, Zhou X and Zhang Y-N 2016 Review on the graphene based optical fiber chemical and biological sensors Sensors Actuators B 231 324-40

[11] Hassan M, Gonzalez E, Hitchins V and Ilev I 2016 Detecting bacteria contamination on medical device surfaces using an integrated fiber-optic mid-infrared spectroscopy sensing method Sensors Actuators B 231 646-54

[12] Xu L, Ge J, Patel J H and Fok M P 2017 Dual-layer orthogonal fiber Bragg grating mesh based soft sensor for 3-dimensional shape sensing Opt. Express 25 24727-34

[13] Woyessa G et al 2017 Zeonex-PMMA microstructured polymer optical FBGs for simultaneous humidity and temperature sensing Opt. Lett. 42 1161-4

[14] Woyessa G, Nielsen K, Stefani A, Markos C and Bang O 2016 Temperature insensitive hysteresis free highly sensitive polymer optical fiber Bragg grating humidity sensor $O p t$. Express 24 1206-13

[15] Urrutia A, Goicoechea J, Ricchiuti A L, Barrera D, Sales S and Arregui F J 2016 Simultaneous measurement of humidity and temperature based on a partially coated optical fiber long period grating Sensors Actuators B 227 135-41

[16] Wang Y, Fu C, Liao C and Wang Y 2016 Refractive index sensing with long period grating in thin-core-fiber Nonlinear Photonics, JM6A 31

[17] Andrews N L et al 2016 In-fiber Mach-Zehnder interferometer for gas refractive index measurements based on a hollowcore photonic crystal fiber Opt. Express 24 14086-99 
[18] Yu X, Gao W, Qu Y and Shu S 2016 The design of Bending long period of photonic crystal fiber grating sensors $O p t$ Photonics J. 6120

[19] Liu X, Jiang M, Sui Q and Geng X 2016 Optical fibre FabryPerot relative humidity sensor based on HCPCF and chitosan film J. Mod. Opt. 63 1668-74

[20] Liu D et al 2016 High sensitivity sol-gel silica coated optical fiber sensor for detection of ammonia in water Opt. Express 24 24179-87

[21] Wu Q, Semenova Y, Wang P and Farrell G 2011 High sensitivity SMS fiber structure based refractometer-analysis and experiment Opt. Express 19 7937-44

[22] Kaczmarek C 2016 Spectral-domain measurement of the strain sensitivity of phase modal birefringence of polarizationmaintaining optical fibers Opt. Commun. 375 43-8

[23] Wang $\mathrm{H}$ et al 2016 Downsized sheath-core conducting fibers for weavable superelastic wires, biosensors, supercapacitors, and strain sensors Adv. Mater. 28 4998-5007

[24] Li M, Liu Y, Gao R, Li Y, Zhao X and Qu S 2016 Ultracompact fiber sensor tip based on liquid polymer-filled Fabry-Perot cavity with high temperature sensitivity Sensors Actuators B 233 496-501

[25] Soltanian M R K et al 2016 Variable waist-diameter MachZehnder tapered-fiber interferometer as humidity and temperature sensor IEEE. Sens. J. 16 5987-92
[26] Li C, Ning T, Zhang C, Wen X, Li J and Zhang C 2016 Liquid level and temperature sensor based on an asymmetrical fiber Mach-Zehnder interferometer combined with a fiber Bragg grating Opt. Commun. 372 196-200

[27] Velázquez-González J S, Monzón-Hernández D, Moreno-Hernández D, Martínez-Piñón F and Hernández-Romano I 2017 Simultaneous measurement of refractive index and temperature using a SPR-based fiber optic sensor Sensors Actuators B 242 912-20

[28] Wang P, Brambilla G, Ding M, Semenova Y, Wu Q and Farrell G 2011 High-sensitivity, evanescent field refractometric sensor based on a tapered, multimode fiber interference Opt. Lett. 36 2233-5

[29] Wang X-D and Wolfbeis O S 2012 Fiber-optic chemical sensors and biosensors (2008-2012) Anal. Chem. 85 487-508

[30] Wu Q, Semenova Y, Hatta A M, Wang P and Farrell G 2010 Bent SMS fibre structure for temperature measurement Electron. Lett. 46 1129-30

[31] Chen F and Wolcott M P 2014 Miscibility studies of paraffin/ polyethylene blends as form-stable phase change materials Eur. Polym. J. 52 44-52

[32] Chen F and Wolcott M P 2015 Polyethylene/paraffin binary composites for phase change material energy storage in building: a morphology, thermal properties and paraffin leakage study Sol. Energy Mater. Sol. Cells 137 79-85 